\title{
Gestimmte Denkräume. Anmerkungen zu Jürgen Hasses „Was Räume mit uns machen - und wir mit ihnen“ (2014)
}

\author{
Simon Runkel \\ Geographisches Institut, Universität Heidelberg, Berliner Straße 48, 69120 Heidelberg, Deutschland \\ Correspondence to: Simon Runkel (simon.runkel@uni-heidelberg.de)
}

Received: 25 January 2017 - Revised: 1 June 2017 - Accepted: 7 June 2017 - Published: 13 July 2017

\begin{abstract}
Kurzfassung. Based on a reading of the book "Was Räume mit uns machen - und wir mit ihnen. Kritische Phänomenologie des Raumes" by Jürgen Hasse (2014), the article discusses the noteworthy role of phenomenology within German-speaking human geography. The phenomenological work by Hasse and his close referring to the philosophy of H. Schmitz will be discussed in the context of the sociology of knowledge and the history of the discipline. In conclusion, the article pleas for a phenomenologically grounded discussion of the spatialities of feelings against the backdrop of the current resurgence of politics of feelings.
\end{abstract}

Denkkollektive werden von Denkstilen zusammengehalten, so eine These Ludwik Flecks (2012:129-145). In vorherrschende Denkstile kontrovers hineinzustoßen erfordert die Fähigkeit, dem Druck dominanter Denkstimmungen (Hasse, 2014:171; 198-199) standzuhalten. Das Buch „Was Räume mit uns machen - und wir mit ihnen. Kritische Phänomenologie des Raumes“ von Jürgen Hasse (2014) gibt zum einen aus wissenssoziologischer Perspektive beredt Zeugnis vom Unternehmen des Autors, einem allzu rationalistischen Paradigma humangeographischer Forschung mit phänomenologischen Ansätzen auf den Leib zu rücken. Zum anderen entfaltet der Autor einen klugen Begriffskosmos lebensphilosophisch inspirierter Raumtheorie, der Anregung zur Würdigung der wichtigen Rolle nicht-szientistischer Ansätze wie den der Phänomenologie für die deutschsprachige Humangeographie bis heute gibt (vgl. Sedlacek, 1989:10).

Das Buch versammelt zwanzig Aufsätze und Vorträge, die der Autor in den Jahren von 2002 bis 2013 verfasst hat. Bis auf eine Ausnahme - „Zum Situationscharakter des Wohnens" (Kapitel 8) - sind alle Beiträge bereits vormalig publiziert und wurden in überarbeiteter Version neu arrangiert. Das Buch vermittelt folglich die thematische Bandbreite des Autors. Es eröffnet einen dezidierten Blick auf die Feinheiten seines schriftstellerischen und wissenschaftlichen Schaffens. Der thematische Umfang der Kompilation macht es schwierig das Buch in seiner erzählerischen Stringenz zu rezensieren. Stattdessen bietet sich die Gelegenheit zur umrisshaf- ten Besprechung der geisteswissenschaftlichen Raumphänomenologie, die in der vor allem deutschsprachigen Humangeographie in den letzten Dekaden in all ihrer notwendigen Streitbarkeit markant mit dem Namen des Autors verbunden war.

Zunächst einige Worte zum Buch selbst: der kompilatorische Charakter des Buchs hat Tücken. Die Rahmung und Verknüpfung der Artikel gelingt vor allem durch ihre gleichsame Verwurzelung in der Phänomenologie, genauer gesagt vor dem Hintergrund des ausgefeilten Systems der Philosophie des Kieler Leibphänomenologen Hermann Schmitz (als sog. Neue Phänomenologie in zehn Bänden von 1964 bis 1980 entwickelt; zur Einführung Schmitz, 2009a). Man kann der Themenauswahl im Buch Eklektizismus vorwerfen: zwischen Architekturkritik (Kapitel 3, 10, 15, 18), Raumtheorie (Kapitel 1, 12, 13), stadtgeographischer Theoriebildung (Kapitel 2, 6, 7, 10, 11, 14) lassen sich kulturkritische Einlassungen zu Ernährung(spraktiken) (Kapitel 4, 5), zum Wohnen (Kapitel 8), anhand von Literatur (Kapitel 9, 17) und Malerei (Kapitel 20) zur geopoetischen Weltbeschreibung, zum Verhältnis von Raum und Religion (Kapitel 16) und zu sepulkralkulturellen Praktiken (Kapitel 19) finden. Dieser thematisch bunte Reigen macht die Lektüre zuweilen sehr überraschend, da die verschiedenen Winkel aus denen sich der Autor dem anthropologischen Verhältnis zum Raum widmet, vor allem durch eine in der Phänomenologie wurzelnden Kulturkritik geklammert ist. 
Die phänomenologische Reflexion gehört spätestens seit der Entwicklung der humanistic geography (zum historischen Kanon gehören dazu Arbeiten von Buttimer, Entrikin, Ley, Pickles, Relph, Seamon und Tuan) in der angelsächsischen Geographie zum methodisch-konzeptionellen Kanon humangeographischer Wissensproduktion. In der deutschsprachigen Geographie wurde im letzten Drittel des 20. Jahrhunderts unter interessierter Annäherung an diese Arbeiten und ihrer phänomenologisch-hermeneutischen Fundierungen - und in viel geringerem Maße auch durch eine eher skeptisch geführte Diskussion der radical geography und ihrer marxistischen Positionen (vgl. u.a. Tzschaschel, 1986:144-152; Belina et al., 2009) - eine kritische Auseinandersetzung mit dem Szientismus geführt (Sedlacek, 1982; Boesch, 1989:121-157). Aus dieser kritischen Bewegung gingen schließlich verschiedene Entwürfe zur Neukonzeption einer (qualitativen) Sozialgeographie hervor. Dazu zählen unter anderem Pohls Rekonstruktionsversuch der Geographie als hermeneutische Wissenschaft (1986), Weichharts phän.-informierte Entwürfe einer handlungstheoretischen Sozialgeographie (1986) und schließlich Werlens bis heute prominente handlungstheoretische Programmatik einer rationalistischen Sozialgeographie (1987). Während sich Werlen (1986) frühzeitig mit Bezug auf Karl Popper gegen das Verstehen subjektiver Sinnkonstitutionen wendete und sich nur bedingt der phänomenologischen Tradition (nämlich v.a. einer Sozialphänomenologie nach Alfred Schütz und dem Lebenswelt-Begriff) anschloss, so schlug Hasse (1989) schließlich ein dezidiert (raum-)phänomenologisches Forschungsprogramm zur kritischen Reflexion postmoderner Wirklichkeiten vor (vgl. Sahr, 1997:30-33). Diese in den 1980ern vollzogene, ,individualtheoretische Wende“ (Sahr, 2003:241) in der deutschsprachigen Sozialgeographie ermöglichte nicht nur Anschlüsse an internationale Entwicklungen, sondern schaffte Grundlagen für die Aufnahme des cultural turn in der Humangeographie, was schließlich in der Bewegung der Neuen Kulturgeographie um die Jahrtausendwende kristallisierte (vgl. Gebhardt et al., 2003). Hasses nahezu im Alleingang durchgeführtes - Projekt einer phänomenologischen Geographie lässt sich vor dem Hintergrund dieser disziplinhistorischen Entwicklungen seit den 1980ern in der Geographie besser verstehen. Es ist konsequent, in seinem Gesamtwerk einen theoretischen Stützpfeiler gegenwärtiger sozial- und kulturgeographischer Ausrichtungen und Entwicklungen zu sehen.

Re-Importe kontinentalphilosophischer Ausrichtungen gehören grundsätzlich zu den Eigentümlichkeiten ,internationalisierter" Denkkollektive in den deutschsprachigen Sozialwissenschaften der letzten Jahrzehnte. Dies schließt selbstverständlich die Übernahme theoretischer Positionen von der angelsächsischen in die deutschsprachige Geographie ${ }^{1}$ mit

\footnotetext{
${ }^{1}$ Selten wurden Ansätze der deutschsprachigen Geographie der angelsächsischen Geographie zugänglich gemacht. Die Münchner Gruppe um Robert Geipel (aus der Jürgen Pohl entstammt) bemüh-
}

ein. In Bezug auf die unterschiedlichen phänomenologischen Ausdifferenzierungen kommt hinzu, dass dies im Kontext der 1970er und 1980er Jahre in der geographischen Disziplin anrüchige Erinnerungen an die ästhetische Landschaftsgeographie weckte (vgl. Hard, 1973:156-159). Ein Re-Import phänomenologischer Denkstile wirkte in diesem Zusammenhang, so lässt sich vermuten, eher unverdächtig und bot damit Anregung zur erneuten Vervielfältigung von Denkstilen. Die kontinentale Phänomenologie wurde im Rahmen der humanistic geography zwar weitreichend rezeptiert und diskutiert (Relph, 1970; Tuan, 1971; Ley und Samuels, 2014 [1978]), aber vor allem im Anschluss an Husserls Phänomenologie und den sozialphänomenologischen Lebenswelt-Ansatz von Schütz (Buttimer, 1976) sowie Heideggers Fundamentalontologie (Pickles, 1985) zugespitzt (zum Überblick siehe Werlen, 1999:213-217; Werlen, 2008:77-78; Dörfler und Rothfuß, 2017). Eben jene Traditionen fanden sich folglich zunächst verstärkt in einer deutschsprachigen Diskussion der Phänomenologie und Sozialgeographie wieder, gleichwohl deutlich ist, dass der sich seit Husserls methodisch konturierter Bewusstseinsphänomenologie - um hier psychologische und philosophische Vorläufer mal auszuklammern - phänomenologische Denkraum weitaus mehr nuancierte Denkstile aufwies, als es die humanistic geography repräsentieren konnte. Dazu gehören ferner phänomenologische Stile rechts- sowie linksrheinischer Façon, beispielsweise von Hans Lipps, Gaston Bachelard, Otto Friedrich Bollnow und vor allem leibzentrierte Phänomenologien von Maurice Merleau-Ponty und Hermann Schmitz.

Hasses phänomenologisches (und lebensphilosophisches) Unternehmen in der Humangeographie zeigte sich stets unbeeindruckt von der selektiven Rezeption der kontinentalen Phänomenologie durch angelsächsische Autor*innen. Er hat neben seiner Vorliebe für Autoren wie Heidegger, Scheler und Bollnow ein bemerkenswertes Gespür für nahezu vergessene phänomenologische, psychologische, ästhetische und architekturtheoretische Literatur vor allem aus der Zeit des ausgehenden 19. Jahrhunderts und der ersten Hälfte des 20. Jahrhunderts. Im Kern jedoch kreist sein konzeptioneller Ansatz um Schmitz' Philosophie, ,dessen Phänomenologie große begriffliche Differenziertheit in der Analyse menschlicher Gefühle erreicht hat und den Vorteil für sich in Anspruch nehmen kann, ontologische Fragen der Gefühle erkenntnistheoretisch systematisch eingebettet zu haben" (Hasse, 2014:83). Schmitz' Phänomenologie stellt die leibliche Erfahrung zentral, von welcher er sämtliche Begriffe und Konzepte wie unter anderem „Situation“, „Halbdinge“, „leiblicher Raum“, „Atmosphären als räumliche ergossene Gefühle“ oder „leibliche Kommunikation“ entwickelt. Dabei entfaltet Schmitz also ausgehend vom Leib einen sehr eigenwilligen Begriffskosmos zur phänomenologischen Beschreibung des affektiven Betroffenseins im Dasein, welcher

te sich aktiv um einen Austausch zu humanistic geographers (vgl. Geipel, 2014 [1978]). 
im direkten Gegensatz zu rationalistischen Menschenbildern und -begriffen steht. Hasse machte sich für seine Arbeiten dieses phänomenologische Vokabular zu eigen und stellt dies ins Licht humangeographischer Frage- und Problemstellungen. Insbesondere lag Hasses Fokus dabei auf den inspirierenden raumphänomenologischen Erörterungen Schmitz', v.a. des Atmosphären-Begriffs, den der Didaktiker und Sozialgeograph für die Stadtforschung und -entwicklung aufarbeiten und für grundsätzliche Fragen der Mensch-UmweltBeziehung klug in Szene setzen konnte. Für die deutschsprachige Geographie entwickelte er somit seit den 1980ern eine begrifflich und konzeptionell recht eigenständige, d.h. von den anfänglichen Impulsen der humanistic geography weitgehend emanzipierte, phänomenologische Denk- und Schreibweise. Das vorliegende Buch gibt einen interessanten Einblick in diese Arbeit. Dass die Betonung gefühliger Weltund Landschaftserfahrung in der deutschsprachigen Humangeographie eine umstrittene Tradition hat, blendet Hasse allerdings aus: ästhetisch-geographische Versuche - vor allem zur ganzheitlich erfahrbaren Landschaft - der ersten Hälfte des 20. Jahrhunderts (bspw. Banse, Granö und einige andere) bleiben im Buch erstaunlicherweise unerwähnt und werden entsprechend weder disziplingeschichtlich problematisiert, noch werden generell historische Kontextualisierungen der zentralen Autoren (wie beispielsweise zu Heidegger) angeboten. Dennoch ist Hasse sich bewusst, dass sich die Phänomenologie nicht gesellschaftspolitischer Zugriffe entziehen kann, weswegen er sich mit in einer mal mehr, mal weniger deutlich konturierten Positionierung im kulturkritischen Kielwasser der „Frankfurter Schule“ verortet. Diesen Bezügen steuert Hasse ferner Verweise auf zeitgenössischere Denker der Postmoderne wie Baudrillard, Foucault oder Lyotard bei.

Die intensive Auseinandersetzung mit Schmitz ist ungewöhnlich. Dessen System der Philosophie wird in der eigenen Disziplin ${ }^{2}$ wenig Aufmerksamkeit entgegengebracht und wenn, dann häufig in eher kritischer Haltung. Um den nunmehr recht betagten Philosophen rankt sich ein kleiner Kreis von interdisziplinären Freund*innen und Förder*innen, die sein Schaffen in direkter Folge des Heidegger'schen Projekts wahrnehmen. Schmitz' Schaffenskraft, seine begriffliche Präzision, seine beeindruckende Beharrlichkeit, den Dingen phänomenologisch nachzuspüren sowie die Dialektik einer bescheidenen Persönlichkeit und dem kühnen Anspruch mit einer „Neuen Phänomenologie“ die europäischen Denkstile (insbesondere noch vor Bacon, Hobbes, Galilei, Descartes und Leibniz zurückzugehen) grundsätzlich zu kritisie-

\footnotetext{
${ }^{2}$ Von einer soziologischen Auseinandersetzung mit der Phänomenologie von Schmitz ganz zu schweigen: in einem prominenten Sammelband zum Verhältnis von Phänomenologie und Soziologie findet Schmitz keinerlei Erwähnung (Raab et al., 2008). Andererseits, so scheint es zumindest, nimmt auch Jürgen Hasse wenig Notiz von aktuellen phänomenologischen Debatten innerhalb der Soziologie.
}

ren, macht ihn attraktiv als Gallionsfigur einer oppositionellen Denkstimmung. Das hat übrigens auch Peter Sloterdijk in seinem Sphären-Projekt erkannt, der zwar zu den flüchtigsten, aber wohl populärsten Schmitz-Rezipienten gehört.

Eines der Ziele Schmitz' ist eine profunde Wissenschaftskritik, die unweigerlich in eine Kultur- und Zivilisationskritik mündet (vgl. einführend dazu Schmitz, 2009b). Adressiert ist sein Vorhaben vor allem an die exakten Naturwissenschaften, welche - im Prinzip seit Demokrit - die unwillkürliche Lebenserfahrung systematisch ausblenden. Aufgrund dieses Reduktionismus, so Schmitz, wurde die Welt in ein Innen und Außen zerteilt. Das Außen wurde abgeschliffen und in identifizierbare, manipulierbare und quantifizierbare Merkmale zerlegt. Dies bildet heute die naturwissenschaftliche Abstraktionsbasis. Der „Rest“" wurde psychologisiert, das heißt in die Innenwelt der Bewussthaber introjiziert durch Konzepte wie Seele, Gemüt, Psyche, Bewusstsein o.ä. Schmitz bietet nun einen Gegenentwurf an, in dem er u.a. in seiner Leib-zentrierten Phänomenologie die Lebenserfahrung, die Räumlichkeit der Gefühle - v.a. vermittels dem Konzept der Atmosphären - und einen ,situationistischen Holismus“ (Hasse, 2014:36) betont. Schmitz (2014a) kritisiert die Unterteilung in eine Innen- und Außenwelt dahingehend, dass er phänomenologisch auf eine leiblich zu spürende ,primitive Gegenwart“ (ebd.:88) verweist, die unspaltbar ist, sich aber - durch die menschliche Fähigkeit zur satzförmigen Rede - vereinzeln, d.h. in „Gattungen nach Übereinstimmungen und Unterschieden“ (ebd.:95) gruppieren lässt. Damit erscheint die Welt als ,entfaltete Gegenwart" (ebd.:96). Diese phänomenologische Entfaltung skizziert Schmitz als einen erkenntnistheoretischen Explikationismus, der sich als dritte Option neben Realismus und Idealismus verstehen lässt.

Es ist Hasse zu verdanken, dass er in dieser leibphänomenologischen Programmatik und ihrem erkenntnistheoretischen Überbau als einer der ersten das kritische Potential für die Humangeographie entdeckt hat. Hasse überträgt die Schmitz'sche Kritik an den Naturwissenschaften auf die Sozialwissenschaften. In eloquenter Weise trägt er sie nun an die seit den 1980er Jahren florierende qualitative Sozial- und Kulturgeographie heran; vor allem an diejenigen Ansätze, die ein rational handelndes Subjekt zum Ausgangspunkt ihrer akteurszentrierten Theorien machen. Hasses phänomenologische Kritik an einem konstruktivistischen Menschenbild (be)trifft dabei vor allem die handlungstheoretische Sozialgeographie von Werlen. Die Feinheiten dieser Anfang der 2000er zur vollen Prominenz gelangenden Menschenbild-Debatte lassen sich an dieser Stelle leider nicht ausgiebig genug reproduzieren (Hasse und Helbrecht, 2003). Der zuweilen missionarisch anmutende Eifer dieses - damalig hochspannenden, notwendigen und scharfsinnigen - Anliegens durchzieht das vorliegende Buch nach wie vor. Zentraler Gegenentwurf zum Akteursbegriff ist für Hasse der Begriff des Patheurs, des emotional Betroffenen (Hasse, 2014:14). Das von ihm als Leitparadigma der Hu- 
mangeographie ausgemachte handlungstheoretische Wissenschaftsverständnis (ebd.:22) ist nach wie vor die Reibungsfläche seiner Kritik. Ferner wird die Sprachzentrierung des linguistischen und konstruktivistischen Menschenbilds einer Kritik unterzogen (ebd.:37).

Diese Kritiken haben ihre Berechtigung. Für diese Pointierungen in den letzten Jahren gebührt Jürgen Hasse Dank. Nicht nur weil er unweigerlich als Wegbereiter für konzeptionelle Umbrüche in der Humangeographie gelesen werden muss, sondern auch vor dem Hintergrund, dass er in den vorangegangenen Dekaden nicht nur zum einsamen Rufer in der Wüste stilisiert wurde, sondern bisweilen in eine Denkstimmung hineinschreiben musste, deren Protagonisten in seinen Werken einen ,biedermeierlichlebenshilfeliterarische[n] warme[n] Dunst" sowie betroffene Melancholie und Bombast identifizierten (Hard, 2000:61). Die Kontroverse wurde mit harten Worten geführt, zum einen da eben aus rationalistischer Perspektive die Rede vom ganzheitlich fühlenden Menschen ungute Erinnerungen an die Landschaftsgeographie der ersten Hälfte des 20. Jahrhunderts hervorrief (ebd.:57) und zum anderen, weil sich Hasse selbst in politisch äußerst bedenkliche und wissenschaftlich verwegene Schreiballianzen begab, die ihn zu Recht angreifbar machten. ${ }^{3}$ Es stellt sich die Frage, ob die Erinnerung an dieses kritische Kreuzfeuer zu den zahlreichen Zwischentönen in Hasses Buch führte, die - mit Verweis auf das Verhältnis von Rationalität und Irrationalität in der Wissenschaft - vom „Anpassungsdruck“ (Hasse, 2014:133) und einer „Geographie der Gewalt" (ebd.:197) in der wissenschaftlichen community erzählen, den Fachkolleg*innen dissoziative Identitätsstörungen unterstellen (ebd.:150), das Unsagbare in diskursiven Gemeinschaften thematisieren und von den Ausgeschlossenen bestimmter aufmerksamkeitsfixierter „Rationalitätsbezirke“ und ,libidinös besetzt[er]“ Theorien (ebd.:201) zu berichten wissen (ebd.:201; 314-315). Man kommt nicht umhin, eine gewisse Verbitterung zu detektieren, die im Lichte gegenwärtiger Debatten zur Renaissance geisteswissenschaftlicher Perspektiven in der Humangeographie etwas nostalgisch scheint. $\mathrm{Zu}$ erwähnen sei hier vor allem eine - un-

\footnotetext{
${ }^{3}$ Angesprochen ist hier die Debatte in der Zeitschrift Erdkunde, in welcher Hasse mit dem rechtsesoterischen Naturphilosophen und Geomantiker Reinhard Falter einen Artikel zu „Landschaftsfotografie und Naturhermeneutik“ (2001) publizierte und verteidigte (2002), der insbesondere Gerhard Hard (2001, 2002) Anlass zu teils im Ton sehr heftigem, aber auch in der Sache sehr scharfsinnigem Widerspruch gab. Hasse stand als Ko-Autor zweifellos in einer weltanschaulichen Mitverantwortung. Ob Hards Angriffe auf persönlicher Ebene notwendig oder ob seine Einschätzungen insb. zur (Neuen) Phänomenologie in dieser Debatte uneingeschränkt treffsicher waren, kann man diskutieren. Wichtiger ist aber, dass seine Kritik die Nähe der - anscheinend eher durch Falter denn Hasse induzierten - Argumentationen zu antidemokratischen Diskursen entlarvt und er somit für die Gefährlichkeit bestimmter Denkwege sensibilisiert.
}

ter anderem aus konstruktiver Verbindung von Feminismus und Phänomenologie gewonnene - Hinwendung zu emotionalen, affektiven und nicht-repräsentationalen Geographien sowie einer zumindest international stark aufstrebenden Atmosphärenforschung (siehe Ambiances Network) oder auch der Popularisierung eines (neo)monadologischen statt streng phänomenologischen (Atmo-)Sphären-Begriffs durch Sloterdijk (vgl. Runkel, 2014, 2017). Schon längst ist Hasses Kritik in der deutschsprachigen Humangeographie auf fruchtbaren Boden gestoßen, was ihm in Teilen durch seinen ihm nicht zu verübelnden Rückzug aus den humangeographischen Denkräumen entgangen zu sein scheint. Es wird ihn aber hoffnungsvoll stimmen, dass eine neue Generation junger Schreiber*innen derzeit die Bleistifte spitzt, um den internationalen Debatten bisher vernachlässigte kontinentalphilosophische Konzepte wieder einzuschreiben und in hermeneutisch-phänomenologischem Ernst das gráphein des Irdischen in den Mittelpunkt der Geographie als leiblich erfahrbare und uns verändernde Wissenschaft zu rücken (vgl. Zahnen, 2012).

Kritisch anzumerken ist nun, dass Hasse dem Vordenker Schmitz wenig von der Seite weicht, gleichwohl er die Neue Phänomenologie durch ein paar kluge Ergänzungen aus geographischer Perspektive bereichert. Ein Versuch, Schmitz' Denkansätze zu kritisieren und dadurch weiterzuentwickeln, lässt sich im vorliegenden Buch an manchen Stellen vermissen. In strategischer Hinsicht stärkt er eine Unterscheidung von Stimmungen und Atmosphären, um so mehr Freiraum für architektur- und stadttheoretische Erörterungen zu gewinnen (Hasse, 2014:253-256). Ferner ist es ein Verdienst Hasses, das phänomenologische Unterfangen mit Fragen der Macht zu verknüpfen und sich so der politisch relevanten Suggestionskraft von Atmosphären zu nähern (ebd.:208). Aktuell und progressiv erscheinen hier seine Überlegungen zur Bioethik (ebd.:117), zur Gouvernementalität (ebd.:260) und zur - einem Begriff Baudrillards folgend - „radikalen Verführung" durch die Kulturindustrie. Gleichwohl entkommt man bei der Lektüre einem zuweilen etwas anstrengend wirkenden moralischen Zeigefinger nur selten. Die hermeneutische Spannung entsteht, so spekuliere ich, durch einen gewissen Elitarismus, der mitunter schon bei Schmitz angelegt ist: Phänomenologie erfordert eine Schulung der Sinne und die Fähigkeit zum ästhetischen Ausdruck gefühlter Wirklichkeiten. Diese (vermeintlich?) notwendige Hochsensibilität und Rezeptibilität gibt der Phänomenologie einen didaktisch-pädagogischen Impetus (der sich möglicherweise auch institutionell verorten lässt). Mit anderen Worten: Hasses Duktus erfordert eine Offenheit für poetischen Ernst auf Seiten der Leser*innen und liest sich nicht immer voraussetzungsfrei. Es wird ferner manchen Leser*innen des Konvoluts ein Steigbügel für das Verständnis der phänomenologischen Methode fehlen.

Der Duktus des Autors hat zudem in seinem lebensphilosophischen Gewand eine zuweilen mystische, irrationale oder gar arkane Note, zumindest dann, wenn man mit einem 
vornehmlich rationalistisch geschulten (ebd.:200) Denkstil liest. Doch das phänomenologische Projekt hat ganz und gar nichts Esoterisches, im Gegenteil, es ist ein Unterfangen, welches Metaphysik für reine Spekulation hält. Eigentlich. So ganz legt sich bei der Lektüre mancher Abschnitte die Aufdringlichkeit der strukturellen Verwandtschaft zur theosophischen, anthroposophischen und theologischen $\mathrm{Li}$ teratur (man denke an Swedenborg, Steiner oder Moltmann) nicht. Sowohl Taylor (2012) als auch Sloterdijk (2009) haben sich der gesellschaftstheoretisch interessanten Aufgabe gewidmet, der konzeptionellen Wanderung des Seelischen ins (halbdingliche) Atmosphärische eine geistesgeschichtliche Trajektorie unterzulegen. Somit ist es auch nicht verwunderlich, dass Hasse, unter säkularer Absicherung, mit offenem Visier ein Interesse an heiligen und sepukralkulturellen Orten entwickelt. In diesen Beschreibungen der numinos-sublimen Orte fällt ein Aspekt auf, von welchen ausgehend sich eine kritische Diskussion der Raumphänomenologie aufspannen lässt.

Hasse stößt in seinen architekturhistorischen Erkundungen kirchlicher Atmosphären auf den Purismus protestantischer Kirchen. Interessanterweise reagiert Hasse hier katholischverschnupft: „Eine abstrakt-symbolistische und intellektualistische Theologie will ihren heiligen Raum allein aus der Teilhabe der Gläubigen am Gemeinschaftserleben der Gemeinde generieren, aus der gemeinsamen Situation der sich versammelnden Personen“ und weiter ,[i]mplizit werden damit Ansprüche an die Förderung der Konstitution von numinosen Atmosphären durch Architektur, Szenographie und Raumgestaltung im weitesten Sinne negiert. Indes stellt sich die Frage, ob und wie sich ein heiliger Raum allein im sozialen Milieu einer Glaubensgemeinschaft atmosphärisch konstituieren soll, wenn Bauformen und -materialien ein solches Zustandekommen aus phänomenologischer Sicht mitunter massiv behindern" (Hasse, 2014:306-307). Erstaunlich an dieser Einlassung ist, dass Hasse für die Konstitution des Atmosphärischen zwingend baulich-materielle Substanz zu benötigen scheint. Zwar ist der Autor sensibel für die vielfältigen sozialen Situationen in Städten (z.B. Kapitel 6, 7, 9) und Situationen des Wohnens (Kapitel 8), dennoch setzt er keine sozialphänomenologischen Schwerpunkte, sondern sucht gerne recht einsame Orte wie Brachen, Parkhäuser, Kirchen und Friedhöfe auf, deren gesellschaftlichen Zusammenhang er zumeist in Anknüpfung an Foucault heterotopologisch, d.h. als normative Gegenplatzierungen erläutert.

Diese Vorliebe zur einsamen Introspektion teilt er zwar mit der raumphänomenologischen Literatur im Allgemeinen, obwohl Schmitz (2014b) selbst auch „kollektive Atmosphären“ in den Blick zu nehmen weiß. Der Patheur ist auch immer Akteur (ebd.:45); das heißt auch, dass sozialphänomenologisch die erlebte Anwesenheit des oder der Anderen zu einem Begriff der Atmosphäre gerinnt, der sich konzeptionell stärker sozialtheoretisch öffnen könnte. Diese Schrittfolge dürfte für die qualitativ forschende Sozialgeographie weiterhin wertvoll sein, erfordert aber unter Umständen methodo- logische und epistemologische Kompromisse. In Hasses Texten, so lässt sich feststellen, werden zwar Anregungen (z.B. zur ,leiblichen Kommunikation“) gegeben, aber das Potential, den Atmosphären-Begriff stärker soziologisch und politikwissenschaftlich voranzutreiben, wird zugunsten architekturtheoretischer und literarischer Gegenstandsbetrachtungen nicht vollkommen ausgeschöpft. Während die ästhetische Theorie Böhmes (1995) die Mach- und Herstellbarkeit der Atmosphären gleichsam einer intentionalen Weltdekoration überbetont, so ist Schmitz in dieser Hinsicht zurückhaltender, gleichwohl er das Wohnen als das Verfügen über Atmosphärisches charakterisiert hat (Schmitz, 2009b:77).

Hasse identifiziert weitere Formen der seduktiven, manipulativen Macht der Atmosphärenproduktion in erlebnisökonomischen und neoliberalen Zusammenhängen und erkennt den politischen Charakter von Atmosphären (Hasse, 2014:216). Die kulturell geprägten Praktiken der Atmosphärengestaltung verlieren sich zuweilen in den Hintergrund einer nahezu naturalisierten Vorstellung des Atmosphärischen. Atmosphären als räumlich ergossene Gefühle werfen aus Sicht einer politisch informierten Geographie aktuell Fragen auf: so titelte das „Philosophie Magazin“ in der zweiten Ausgabe im Jahr 2017 zum einen „Sie ist wieder da. Die Frage nach der Identität", während zum anderen ein Interview mit Schmitz im Heft mit dem Zitat „Gefühle sind keine Privatsache" angekündigt wurde. Diese thematische Gleichzeitigkeit des (populär-)philosophischen Interesses vor dem Hintergrund gesellschaftspolitischer Entwicklungen muss nicht unbedingt ein Zufall sein: Welche Rolle spielen kollektive, öffentliche Gefühle in der gegenwärtigen Renaissance der (raumbezogenen) Identität? Derzeit lässt sich gesellschaftspolitisch die Rückkehr einer gefühlsgeleiteten Politik beobachten. Politiken, die sich nicht nur von Stimmungen leiten lassen, sondern diese auch selber zu manipulativen Zwecken produzieren, haben oft zum Ziel, das dumpfe emotionale Produkt der „Identität“ zu stiften. Setzt man nun fundamentalontologisch und raumphänomenologisch mit Heidegger - dessen Denken durchaus „giftig“ sein kann (Korf, 2014) - und Schmitz die (vermeintlich) nichtsubjektive Autorität gefühlsräumlicher Ergriffenheit in den Fokus, dann kommt man nicht umhin, raumbezogene Identität auch im Lichte aktueller rechtspopulistischer Instrumentalisierungen des Gefühls zu sehen. Hasses kritische Phänomenologie des Raumes gibt hier wertvolle Hinweise, wenn er von den „subversive[n] Machtausübungen über die kollektiven Gefühle in einer Gesellschaft" (Hasse, 2014:41) und über die politische Kommunikation emotionaler Regungen (ebd.:211) räsoniert.

Gefühle zum Mittelpunkt humangeographischer Überlegungen $\mathrm{zu}$ machen ist nach wie vor ein dringendes, aber zwangsläufig politisches Projekt. Es ist Hasses Verdienst, hier einen Zugang gelegt zu haben. Allerdings bedarf es in paralleler Aktion dazu immer auch kritischer, disziplinhistorischer Aufarbeitungen der Denkstimmungen im humangeographischen Landschaftsparadigma, um bestimmte theoretische Reflexe der damaligen Zeit nicht zu wieder- 
holen. Es erscheint für eine politisch informierte Humangeographie dringlich, diese Aufgabe in kritischer Reflexion des geistesgeschichtlichen Quellgebiets der Phänomenologie und Lebensphilosophie aufzunehmen. Die - zugegebenermaßen strittige - Beobachtung Georg Lukács' (1974:100), dass dem Faschismus ,eine bestimmte politische Atmosphäre, ein Zersetzen des Vertrauens in Verstand und Vernunft, eine Zerstörung des Glaubens an den Fortschritt, eine Leichtgläubigkeit gegenüber Irrationalismus, Mythos und Mystik“ vorauslief, sollte dennoch nicht nur Warnung vor bestimmten Denkzwängen sein, sondern ebenfalls Ausgangspunkt für die Erschließung neuer Denkräume. Hasses Arbeiten sind für diese Aufgaben uneingeschränkt zu empfehlen, denn, wie Fleck (2012:189) schreibt, ,gute, stilvolle Arbeiten erwecken sofort entsprechende solidarische Stimmung beim Leser, und sie ist es, die nach einigen Sätzen das Buch zu schätzen zwingt und wirkungsvoll macht". Dies gilt für Hasses Buch „Was Räume mit uns machen - und wir mit ihnen“ uneingeschränkt. Man kann sein wissenschaftliches Anliegen somit als erfolgreich verstehen, da es bleibenden Wert hat (vgl. ebd.:130). Es nun mit wohlwollender Solidarität und in distanzierter Reflexion des ,inter- und intrakollektiven Denkverkehrs“ (ebd.:139-145) weiterzuentwickeln erfordert neue, kritische Leser*innen.

Datenverfügbarkeit. Für diesen Artikel wurden keine Datensätze genutzt.

Interessenkonflikt. Die Autoren erklären, dass kein Interessenkonflikt besteht.

Danksagung. Mein Dank geht an Hans Gebhardt, Benedikt Korf als Herausgeber und eine*n Gutachter*in für wertvolle Anregungen.

Edited by: Benedikt Korf

Reviewed by: one anonymous referee

\section{Literatur}

Belina, B., Best, U. und Naumann, T.: Critical geography in Germany: from exclusion to inclusion via internationalisation, Social Geography, 4, 47-58, 2009.

Boesch, M.: Engagierte Geographie. Zur Rekonstruktion der Raumwissenschaft als Politik-orientierte Geographie, (= Erdkundliches Wissen, H. 98), Franz Steiner Verlag, Stuttgart, 1989.

Böhme, G.: Atmosphäre. Essays zur neuen Ästhetik, Suhrkamp, Frankfurt am Main, 1995.

Buttimer, A.: Grasping the Dynamism of Lifeworld, Ann. Assoc. Am. Geogr., 66, 277-292, 1976.

Dörfler, T. und Rothfuß, E.: Place, Life-World and the Leib: A Reconstructive Perspective on Spatial Experiences in Human Geography, in: Place, Space and Hermeneutics, (= Contributions to Hermeneutics, Vol. 5), Herausgeber: Janz, B. B., New York, Springer, 413-425, 2017.

Falter, R. und Hasse, J.: Landschaftsfotografie und Naturhermeneutik. Zur Ästhetik erlebter und dargesteller Natur, Erdkunde, 55, 121-137, 2001.

Falter, R. und Hasse, J.: Geographie und das Mensch-NaturVerhältnis, Erdkunde, 56, 81-94, 2002.

Fleck, L.: Entstehung und Entwicklung einer wissenschaftlichen Tatsache, Suhrkamp, Frankfurt am Main, 2012.

Gebhardt, H., Reuber, P., und Wolkersdorfer, G. (Hrsg.): Kulturgeographie. Aktuelle Ansätze und Entwicklungen. Spektrum Akademischer Verlag, Heidelberg, 2003.

Geipel, R.: The landscape indicators school in German geography, in: Humanistic Geography. Prospects and Problems, (= Routledge Library Editions: Social and Cultural Geography, Vol. 15), Herausgeber: Ley, D. und Samuels, M. S., Routledge, London und New York, 155-172, 2014 [1978].

Hard, G.: Die Geographie. Eine wissenschaftstheoretische Einführung, Walter de Gruyter, Berlin, 1973.

Hard, G.: Von melancholischer Geographie, Geographische Revue, 2/2000, 39-66, 2000 .

Hard, G.: „Hagia Chora“. Von einem neuerdings wieder erhobenen geomantischen Ton in der Geographie, Erdkunde, 55, 172-198, 2001.

Hard, G.: Eine einfältige Erzählung. Zu Falters und Hasses Text über „Die Geographie und das Mensch-Natur-Verhältnis“, Erdkunde, 56, 95-104, 2002.

Hasse, J.: Sozialgeographie an der Schwelle zur Postmoderne. Für eine ganzheitliche Sicht jenseits wissenschaftstheoretischer Fixierungen, Zeitschrift für Wirtschaftsgeographie, 33, 20-39, 1989.

Hasse, J.: Was Räume mit uns machen - und wir mit ihnen, Kritische Phänomenologie des Raumes, Freiburg und München, 2014

Hasse, J. und Helbrecht, I.: Menschenbilder in der Humangeographie, (= Wahrnehmungsgeographische Studien, Bd. 21), BIS, Oldenburg, 2003.

Korf, B.: Critical geography and the poison of Heidegger's thought, Geogr. Helv., 69, 145-146, https://doi.org/10.5194/gh-69-1452014, 2014.

Ley, D. und Samuels, M. S. (Hrsg.): Humanistic Geography. Prospects and Problems, (= Routledge Library Editions: Social and Cultural Geography, Vol. 15), Routledge, London und New York, 2014 [1978].

Lukács, G.: Die Zerstörung der Vernunft. Band II, Irrationalismus und Imperialismus, Luchterhand, Darmstadt, 1974.

Philosophie Magazin, 02/2017, Februar März, Berlin, 2017.

Pickles, J.: Phenomenology, science and geography, Spatiality and the human sciences, Cambridge University Press, Cambridge, 1985.

Pohl, J.: Geographie als hermeneutische Wissenschaft. Ein Rekonstruktionsversuch, (= Münchener Geographische Hefte, Nr. 52), Verlag Michael Lassleben, Kallmünz/Regensburg, 1986.

Raab, J., Pfadenhauer, M., Stegmaier, P., Dreher, J., und Schnettler, B. (Hrsg.): Phänomenologie und Soziologie. Theoretische Positionen, aktuelle Problemfelder und empirische Umsetzungen, VS Verlag für Sozialwissenschaften, Wiesbaden, 2008.

Relph, E.: An inquiry into the relations between phenomenology and geography, Canadian Geographer, 14, 193-201, 1970. 
Runkel, S.: Sicherheit für die feiernden Massen: Sloterdijks Schaumtheorie und die interorganisationale Umsetzung von Großveranstaltungsprojekten, Berichte, Geographie und Landeskunde, 88, 317-336, 2014.

Runkel, S.: Monadologie und Sozialgeographie, Theoretische Perspektiven mit Gabriel Tarde, Geographische Zeitschrift, 105, 5275, 2017.

Sahr, W.-D.: Ville \& Countryside - Land-Stadt-Verflechtungen im ländlichen St. Lucia. Ein Beitrag zu einer postmodernen Sozialgeographie der Karibik, Wayasbah, Hamburg, 1997.

Sahr, W.-D.: Der Cultural Turn in der Geographie, in: Kulturgeographie. Aktuelle Ansätze und Entwicklungen, herausgegeben von: Gebhardt, H., Reuber, P. und Wolkersdorfer, G., Spektrum Akademischer Verlag, Heidelberg, 231-249, 2003.

Sedlacek, P. (Hrsg.): Kultur-/Sozialgeographie, UTB-Schöningh, Paderborn, 1982.

Sedlacek, P.: Qualitative Sozialgeographie. Versuch einer Standortbestimmung, in: Programm und Praxis qualitativer Sozialgeographie, (= Wahrnehmungsgeographische Studien, Bd. 6), herausgegeben von: Sedlacek, P., BIS, Oldenburg, 1989.

Schmitz, H.: Kurze Einführung in die Neue Phänomenologie, Verlag Karl Alber, Freiburg und München, 2009a.

Schmitz, H.: Der Leib, der Raum und die Gefühle, Aisthesis Verlag, Bielefeld und Basel, 2009b.

Schmitz, H.: Gibt es die Welt?, Verlag Karl Alber, Freiburg und München, 2014a
Schmitz, H.: Atmosphären, Verlag Karl Alber, Freiburg und München, 2014b.

Sloterdijk, P.: Du musst dein Leben ändern, Über Anthropotechnik, Suhrkamp, 2009.

Taylor, C.: Ein säkulares Zeitalter, Suhrkamp, Frankfurt am Main, 2012.

Tuan, Y.-F.: Geography, phenomenology, and the study of human nature, Can. Geogr., 15, 181-192, 1971.

Tzschaschel, S.: Geographische Forschung auf der Individualebene. Darstellung und Kritik der Mikrogeographie, (= Münchener Geographische Hefte, Nr. 53), Verlag Michael Lassleben, Kallmünz/Regensburg, 1986.

Werlen, B.: Thesen zur handlungstheoretischen Neuorientierung sozialgeographischer Forschung, Geogr. Helv., 41, 67-76, https://doi.org/10.5194/gh-41-67-1986, 1986.

Werlen, B.: Gesellschaft, Handlung und Raum. Grundlagen handlungstheoretischer Sozialgeographie, (= Erdkundliches Wissen, H. 89), Franz Steiner Verlag, Stuttgart, 1987.

Werlen, B.: Zur Ontologie von Gesellschaft und Raum. Sozialgeographie alltäglicher Regionalisierungen, Band 1, Franz Steiner Verlag, Stuttgart, 1999.

Werlen, B.: Sozialgeographie, Eine Einführung, HauptVerlag/UTB, Bern, 2008.

Zahnen, B.: Kollektiv Erdbewohner. Das geographische Wir, Zeitschrift für Medien- und Kulturforschung, 2, 167-184, 2012. 